\title{
Formulación de hamburguesa gourmet precocida-congelada, usando carne caprina, perejil (Petroselinum crispum) y albahaca \\ (Ocimum basilicum)
}

\author{
Formulación de hamburguesa gourmet precocida- \\ congelada, usando carne caprina, perejil \\ (Petroselinum crispum) y albahaca (Ocimum basilicum)
}

Leila Águeda Prías Mogro Universidad de Guayaquil (Guayaquil - Ecuador) leila.priasm@ug.edu.ec

Raúl Díaz Torres Universidad de Guayaquil (Guayaquil - Ecuador)

Cynthia Mera Morales Universidad de Guayaquil (Guayaquil - Ecuador)

Revista Cumbres Vol.3 №2

Versión impresa ISSN 1390-9541

Versión electrónica ISSN 1390-3365

http://investigacion.utmachala.edu.ec/revistas/index.php/Cumbres 


\section{RESUMEN}

Recientemente se ha visto una creciente demanda de productos cárnicos saludables. Numerosos ingredientes han sido usados para reformular productos cárnicos y promover la presencia de compuestos beneficiosos para la salud. El objetivo de este trabajo fue desarrollar una formulación de hamburguesa a base de carne de ganado caprino, empleando saborizantes naturales con propiedades antioxidantes. Se utilizó un diseño factorial 32 para definir 9 formulaciones de hamburguesa basadas en carne caprina, con diferentes adiciones de perejil (Petroselinum crispum) y albahaca (Ocimum basilicum), comparando sus indicadores de calidad con los establecidos en las normativas ecuatorianas. Se evaluó la composición química de los productos, su calidad higiénica, y el Índice de Calidad Sensorial (ICS) obtenido a partir de la aplicación de una escala hedónica por atributos a 80 consumidores potenciales del producto, así como su índice de costo. Los resultados fueron procesados mediante el paquete estadístico SPSS. La carne utilizada fue Tipo I, de acuerdo a las normativas ecuatorianas. Todas las formulaciones corresponden con la categoría "Me gusta" aunque la formulación 2 (1,5 \% de perejil y 2,5 \% de albahaca) presentó los mayores valores en todos los atributos, especialmente en cuanto a olor y consistencia. Las muestras cumplen con los requisitos microbiológicos establecidos según las normativas ecuatorianas, con valores de aerobios mesófilos, presencia de Escherichia coli y Staphylococcus aureus, inferiores a los permisibles y ausencia de Salmonella, con un índice de costo de 9,57 dólares/kg. Se obtuvo un producto aceptable para el consumidor, inocuo y de alto valor nutricional.

Palabras clave: carne caprina, hamburguesa, calidad, evaluación sensorial

\section{ABSTRACT}

There has recently been a growing demand for healthy meat products. Numerous ingredients have been used to reformulate meat products and promote the presence of beneficial compounds for health. The objective was to develop a hamburger formulation based on goat meat using natural flavorings with antioxidant properties. A factorial design 32 was used to define 9 hamburger formulations based on goat meat with different additions of parsley (Petroselinum crispum) and basil (Ocimum basilicum), comparing their quality indicators with those established in the Ecuadorian regulations. The chemical composition of the products, their hygienic quality, and the Sensory Quality Index (SCI) obtained from the application of a hedonic scale by attributes to 80 potential consumers of the product, as well as their cost index, were evaluated. The results were processed using the SPSS statistical software. The meat used was Type I, in accordance with the Ecuadorian regulations. All formulations correspond to the "Like" category, although formulation 2 (1.5\% parsley and $2.5 \%$ basil) presented the highest values in all attributes, especially in terms of odor and consistency. The samples comply with the Microbiological Requirements established according to the Ecuadorian regu- 
lations, with values of aerobic mesophylls, presence of Escherichia coli and Staphylococcus aureus, lower than permissible and absence of Salmonella, with a cost index of 9.57 dollars $/ \mathrm{kg}$. A product acceptable to the consumer was obtained, safe and of high nutritional value.

Keywords: goat meat, hamburger, quality, sensory evaluation

\section{INTRODUCCIÓN}

Para alimentar al mundo, el sector agrícola tiene el desafío de aumentar la producción en más del 60\% para el año 2050 (FAO, 2012). En este escenario, se espera que el consumo de carne, fuente fundamental de proteína para la dieta humana, crezca sustancialmente. Aunque la demanda proyectada demuestra que la posición de liderazgo le corresponderá a la carne de pollo y cerdo, seguida por la carne de bovinos y ovinos, respectivamente (Montossi et al, 2013), la carne de cabra también tiene su espacio en esta demanda, tomando en consideración que es una especie muy utilizada por el hombre, debido a su carne, leche y piel, así como otras ventajas relacionadas con el control de los matorrales (Aréchiga et al, 2008)

La carne magra de cabra es baja en ácidos grasos saturados y grasas, pero rica en ácidos grasos insaturados, con propiedades hipocolesterolémicas demostradas. Los cortes de carne de cabra tienen niveles de proteína comparable a cortes similares de carne de vacuno o cordero, pero con menor contenido de grasa, y menor porcentaje de grasa saturada las carnes de pollo, res, cerdo, o cordero. Teniendo en cuenta su alto valor nutritivo y su mayor relación de ácidos grasos insaturados / saturados, la carne de cabra tiene el potencial de mejorar la salud de los consumidores manteniendo productos a base de carne en la dieta diaria. Por otra parte, su consumo se está volviendo popular y se encuentra a menudo disponible en el nivel de la alta cocina (Malekian et al, 2016). Sin embargo, factores como la edad del animal (Das et al, 2012; Lopes, 2014) o su alimentación, pueden incidir en la calidad de la carne (Ilavarasan et al, 2015). La carne de cabra presenta un alto contenido de proteína, aunque el porcentaje depende de la raza, con un alto valor biológico de aproximadamente 60,4 y un coeficiente de digestibilidad del 97\%, datos que refuerzan la idea del valor de su consumo (Webb, 2014). Por estas razones, la incorporación de carne caprina en la elaboración de productos cárnicos ha aumentado en los últimos años, donde se reporta su empleo en la elaboración de hamburguesas (Das et al, 2012; Jalal et al, 2015), nuggets (Banerjee et al 2012), embutidos (Malekian et al, 2014; Leite et al, 2015) y rollitos de carne (Yadav et al, 2014). El contenido de grasa de estos productos se relaciona con sus características sensoriales, especialmente las texturales, en términos generales un mayor contenido de grasa permite obtener productos más jugosos, pero con mayor tendencia a la rancidez. (Das, \& Rajkumar, 2013).

El objetivo de este trabajo fue desarrollar una formulación de hamburguesa a base de carne de ganado caprino, empleando antioxidantes naturales como saborizantes y a la vez, prevenir la rancidez. 


\section{MATERIALES Y MÉTODOS}

Formulaciones: todos los ingredientes fueron adquiridos frescos en el mercado local. La carne de cabra, proveniente de los muslos, fue conservada entre $0^{\circ} \mathrm{C}$ y $4^{\circ} \mathrm{C}$, por no más de 24 horas, hasta su utilización para la elaboración de las hamburguesas.

Para la formulación se estableció un diseño factorial 32 (Tabla 1) donde se variaron los niveles de albahaca y perejil y se empleó $6 \%$ de huevo entero y $2,1 \%$ de sal, siendo el resto carne caprina molida. Los ingredientes se mezclaron y amasaron en un recipiente de acero inoxidable, hasta obtener la consistencia esperada y se elaboraron formas planas circulares, de aproximadamente $1 \mathrm{~cm}$ de altura x $8 \mathrm{~cm}$ de diámetro. Las hamburguesas se escaldaron con vapor de agua $\left(100^{\circ} \mathrm{C}\right)$ a una altura de $15 \mathrm{~cm}$, durante $10 \mathrm{~min}$. y se colocaron de forma separada en una bandeja plana de acero inoxidable hasta alcanzar la temperatura ambiente para preservarlas en refrigeración $\left(4^{\circ} \mathrm{C}\right)$ por no más de 24 horas, cubiertas con láminas plásticas flexibles, para evitar la desecación.

\begin{tabular}{|l|l|l|}
\hline \multicolumn{3}{|l}{ Tabla 1. Diseño experimental } \\
\hline FÓRMULA & PEREJIL (\%) & $\begin{array}{l}\text { A L B A H A C A } \\
(\%)\end{array}$ \\
\hline F1 & 1,50 & 1,50 \\
\hline F2 & 1,50 & 2,50 \\
\hline F3 & 1,50 & 3,50 \\
\hline F4 & 2,50 & 1,50 \\
\hline F5 & 2,50 & 2,50 \\
\hline F6 & 2,50 & 3,50 \\
\hline F7 & 3,50 & 1,50 \\
\hline F8 & 3,50 & 2,50 \\
\hline F9 & 3,50 & 3,50 \\
\hline
\end{tabular}

Se evaluó la composición química de los productos obtenidos, su calidad higiénica, y el valor del Índice de Calidad Sensorial (ICS) obtenido a partir de la aplicación de una escala hedónica por atributos a 80 consumidores potenciales del producto, así como el índice de costo de materias primas.

Composición química: se determinó el contenido de nitrógeno (método Kjeldahl) empleando un equipo Kjeldahl Laboratory Company, modelo 3390; contenido total de grasa (Método Soxhlet) utilizando un equipo de extracción de Soxhlet (Precision Scientific Inc, modelo 10-T-2); cenizas totales (mufla, equipo Veb Elektro, Bad Frankenhausen); contenido de humedad escogiendo el escalón 4 en la balanza infrarroja (Mettler Toledo LP11-P160N), el tiempo de secado fue de $11 \mathrm{~min}$. a una temperatura de $115{ }^{\circ} \mathrm{C}$. (NTE INEN-ISO 937:2013-09).

Evaluación sensorial: Para los análisis sensoriales se conformaron dos paneles, uno integrado por 80 consumidores potenciales del producto y el otro integrado por 9 jueces conocedores del producto los que fueron adiestrados en el uso de las técnicas descriptivas.

Las hamburguesas se cocinaron hasta alcanzar una temperatura interna

\section{Cumbres}


de $70{ }^{\circ} \mathrm{C}$ y se cortaron en fracciones de aproximadamente $40 \mathrm{~g}$. Todas las muestras fueron codificadas mediante un código aleatorio de tres cifras. A los consumidores se les presentó una boleta de evaluación donde se les pedía realizar una evaluación hedónica de los atributos sensoriales del producto mediante una escala de cinco categorías, otorgando el valor 5 a la calificación "Me gusta mucho” y el valor 1 a la calificación "Me disgusta mucho”. Los atributos evaluados fueron: Aspecto, Color, Olor, Sabor, Consistencia y Jugosidad. Al grupo de consumidores potenciales se les solicitó ordenar los atributos evaluados según el criterio de importancia de cada uno, para con ello definir un índice de calidad sensorial (ICS) aplicando la formula $\mathrm{ICS}=\mathrm{f} 1(\mathrm{XMaspecto})+\mathrm{f} 2(\mathrm{XMconsistencia})+\mathrm{f} 3(\mathrm{XMcolor})+\mathrm{f} 4(\mathrm{XMolor})+\mathrm{f} 5 \quad$ (XMsabor) $+\mathrm{f} 6$ (XMjugosidad) (Ec1)

Donde f1 a f6 representan el valor del coeficiente de ponderación para cada atributo, obtenido de dividir la puntuación por lugar de cada atributo entre el total de puntuaciones posibles y XM representa el valor medio otorgado por los jueces al evaluar el atributo.

A los jueces adiestrados se les solicitó durante su entrenamiento definir los atributos a evaluar y posteriormente realizar un análisis descriptivo de la hamburguesa de mayor ICS, evaluando dichos atributos mediante una escala grafica no estructurada. Los atributos de este producto fueron comparados con los de tres marcas de hamburguesas del mercado local.

Índice de costos: se determinó para un kilogramo de la fórmula de mayor ICS, multiplicando el costo de un kilogramo de cada materias prima por su fracción en fórmula.

Análisis estadísticos: Se utilizó el programa IBM SPSS Statistics 22. Los datos obtenidos mediante la prueba hedónica fueron transformados a valores numéricos para determinar la media. Se realizó un análisis de varianza para determinar las diferencias entre fórmulas y atributos. En caso de ser necesario se realizó la prueba de Rangos Múltiples de Duncan.

Análisis microbiológicos: se realizaron siguiendo los requisitos microbiológicos para productos cárnicos precocidos congelados (NTE INEN 1338:2012). Para los análisis microbiológicos se pesaron $10 \mathrm{~g}$ de hamburguesa y se homogeneizaron en $90 \mathrm{ml}$ de agua peptonada, Posteriormente, a partir del homogeneizado que constituyó la primera dilución, se prepararon las diluciones necesarias para la determinación de aerobios mesófilos (norma NTE INEN 1529-5:2006), Escherichia coli (norma AOAC 991.14 ) y Staphylococcus aureus (norma NTE INEN 1529-14). Para el aislamiento e identificación del género Salmonella, se pesaron $25 \mathrm{~g}$ de muestra, y se homogeneizaron en $225 \mathrm{~mL}$ de caldo lactosado, incubando por 24 horas a $35^{\circ} \mathrm{C}$ (pre-enriquecimiento). $1 \mathrm{~mL}$ de la muestra pre-enriquecida fue transferido a tubos con $9 \mathrm{~mL}$ de caldo Rappaport y caldo selenito F y se incubaron 24 horas a $35^{\circ} \mathrm{C}$. A partir de aquí, se inocularon los medios selectivos y se incubaron a $35^{\circ} \mathrm{C}$ por 24-48 horas de acuerdo a la norma NTE INEN 1529-15. 


\section{RESULTADOS Y DISCUSIÓN}

Caracterización de la carne caprina empleada: según los análisis efectuados la carne empleada presenta $25,47 \pm 1,35 \%$ de proteína, 3,45 \pm 0,35 $\%$ de grasa, $67,45 \pm 0,47 \%$ de humedad y 0,97 $\pm 0,02 \%$ de cenizas. Estos resultados coinciden en general con los reportados por otros autores (Lopes, 2014; Ilavarasan, 2015), aunque son ligeramente inferiores en cuanto al porcentaje de grasa a los valores reportados por Webb (2014). Debe considerarse que esto obedece a que factores como la raza, tipo de alimentación, musculo considerado o edad, entre otros, pueden afectar la composición química de la carne. Los resultados indican que la carne empleada cumple los requisitos físico-químicos según la norma NTE INEN 1 346:2010 y por su contenido en grasa se cataloga como TIPO I o magra, por tener menos del 15\% de grasa (INEN, 2010).

Prueba hedónica: Para determinar los valores de los factores de ponderación f1 a f6 se contabilizó el total de la puntuación emitida por los jueces para cada atributo y se calculó el máximo valor posible para la suma de ordenamiento de los atributos $(\mathrm{T})$ mediante la formula

$$
\mathrm{T}=80(21)=1680(\text { Ec } 2)
$$

Donde 80 es el número de consumidores encuestados y 21 es la sumatoria de los seis lugares que cada persona puede otorgar a los atributos $(6+5+4+3+2+1=21)$.

Tabla 2. Valores acumulados de las frecuencias y valor del factor de ponderación (fi).

\begin{tabular}{|l|l|l|l|l|l|l|}
\hline & ASPECTO & COLOR & OLOR & SABOR & CONSISTENCIA & JUGOSIDAD \\
\hline$\sum 80$ Jueces & 449 & 350 & 327 & 249 & 195 & 110 \\
\hline fi $\left.=\sum 80 / T\right)$ & 0,27 & 0,21 & 0,19 & 0,15 & 0,12 & 0,07
\end{tabular}

Para la industria alimentaria es de gran importancia entender la relación existente entre las propiedades y los atributos de calidad percibidos. Este conocimiento permitiría incorporar al producto final los atributos de calidad deseados mediante la gestión de los procesos a lo largo de la cadena alimentaria (Prieto et al, 2008). La importancia relativa de cada componente de la calidad depende del producto y de los intereses individuales de las personas. Como puede apreciarse, para este producto el atributo más valorado por los jueces fue Aspecto, seguido por Color y Olor, mientras que Jugosidad fue el atributo menos valorado.

Prueba de aceptación: La tabla 3 muestra los valores medios de los resultados de la prueba de aceptación de las nueve formulaciones elaboradas, así como el valor medio del ICS.

Los consumidores calificaron la mayoría de los atributos como "Me gusta", de acuerdo al sistema empleado. Ninguno de los atributos fue valorado con la máxima calificación ("Me gusta mucho") y solo el atributo Jugosidad (fórmula 1) alcanzó la evaluación "Me es indiferente", lo que indica que todos los productos se mantuvieron en la zona de aceptación. Todas las fórmulas presentan un ICS equivalente a "Me gusta", aunque la fórmula de mayor 
Tabla 3. Resultados de la prueba de aceptación

\begin{tabular}{|c|c|c|c|c|c|c|c|c|c|}
\hline \multirow[t]{2}{*}{ ATRIBUTOS } & \multicolumn{9}{|c|}{ FÓRMULA } \\
\hline & $\mathrm{F} 1$ & $\mathrm{~F} 2$ & F3 & F4 & F5 & F6 & F7 & F8 & F9 \\
\hline Aspecto & 4,2 & 4,3 & 4,4 & 4,1 & 4,0 & 4,1 & 3,9 & 3,8 & 4,4 \\
\hline Consistencia & 4,0 & 4,4 & 4,1 & 4,4 & 3,9 & 4,0 & 4,2 & 4,1 & 4,0 \\
\hline Color & 4,1 & 4,1 & 4,1 & 4,2 & 3,9 & 3,9 & 4,1 & 4,1 & 4,1 \\
\hline Sabor & 4,3 & 4,4 & 4,2 & 4,0 & 3,8 & 4,2 & 4,1 & 4,3 & 4,0 \\
\hline Jugosidad & 3,4 & 4,1 & 3,7 & 3,5 & 3,6 & 4,0 & 4,0 & 4,0 & 3,7 \\
\hline Olor & 4,5 & 4,5 & 3,8 & 3,5 & 3,8 & 4,2 & 4,2 & 4,0 & 4,1 \\
\hline ICS & 4,07 & 4,28 & 4,11 & 4,05 & 3,86 & 4,06 & 4,05 & 4,08 & 4,11 \\
\hline
\end{tabular}

1: "Me disgusta mucho"; 5 "Me gusta mucho"

puntuación fue la F2, cuyo espacio sensorial abarca prácticamente el espacio sensorial de todas las formulaciones. La hamburguesa F5 posee la menor puntuación, alejada del valor máximo.

Prueba con jueces adiestrados: Tras varias sesiones de entrenamiento, los jueces, por consenso definieron los atributos sensoriales a evaluar. Los atributos seleccionados fueron cuatro relacionados con la apariencia, dos con el olor, tres con el sabor y cinco con la textura. Una vez definidos los atributos sensoriales a evaluar, se realizó el análisis descriptivo de la hamburguesa caprina seleccionada (F2) y de tres productos del mercado, correspondientes a tres marcas diferentes, seleccionadas entre las marcas líderes del mercado. La tabla 4 presenta los resultados del análisis descriptivo.

Tabla 4. Resultados del análisis descriptivo

\begin{tabular}{|c|c|c|c|c|c|}
\hline ATRIBUTO & MUESTRAS & EMPRESA 1 & EMPRESA 2 & EMPRESA 3 & F2 \\
\hline \multirow{4}{*}{ Apariencia } & Característico & $8,7 \mathrm{ab}$ & $7,9 a b$ & $6,1 \mathrm{a}$ & $9,2 \mathrm{ab}$ \\
\hline & Homogéneo & $8,9 \mathrm{ab}$ & $8,1 \mathrm{ab}$ & $6,9 \mathrm{a}$ & $9,8 \mathrm{ab}$ \\
\hline & Compacto & $8,6 \mathrm{a}$ & 9,4 a & $6,9 \mathrm{a}$ & 8,6 a \\
\hline & Color Café & $4,8 \mathrm{a}$ & $5,8 \mathrm{a}$ & $5,3 \mathrm{a}$ & 5,6 a \\
\hline \multirow{2}{*}{ Olor } & Olor Carne & $5,1 \mathrm{a}$ & $5,3 \mathrm{a}$ & $5,2 \mathrm{a}$ & 5,9 a \\
\hline & Olor Ahumado & 3,6 a & 3,1 a & $1,0 \mathrm{~b}$ & 3,9 a \\
\hline \multirow{3}{*}{ Sabor } & Sabor Salado & $4,8 \mathrm{a}$ & $4,3 \mathrm{a}$ & $3,7 \mathrm{a}$ & $3,7 \mathrm{a}$ \\
\hline & Sabor Carne & $6,1 \mathrm{a}$ & $6,6 \mathrm{a}$ & $5,0 \mathrm{a}$ & $6,7 \mathrm{a}$ \\
\hline & Sabor Picante & $2,0 \mathrm{a}$ & $2,8 \mathrm{a}$ & $1,9 \mathrm{a}$ & 1,9 a \\
\hline \multirow{5}{*}{ Textura } & Consistencia & $4,0 \mathrm{a}$ & $7,3 \mathrm{~b}$ & $5,2 \mathrm{a}$ & 3,4 a \\
\hline & Cohesividad & $5,1 \mathrm{a}$ & 6,9 a & 5,3 a & $5,1 \mathrm{a}$ \\
\hline & Jugoso & $4,4 \mathrm{a}$ & $4,4 \mathrm{a}$ & $3,2 \mathrm{a}$ & $4,9 \mathrm{a}$ \\
\hline & Grasoso & $4,8 \mathrm{~b}$ & $5,0 \mathrm{~b}$ & $1,2 \mathrm{a}$ & $4,0 \mathrm{~b}$ \\
\hline & Masticable & 6,3 a & 6,4 a & $4,8 \mathrm{a}$ & $5,1 \mathrm{a}$ \\
\hline
\end{tabular}

Fórmulas con letras diferentes en la misma fila difieren significativamente $(p<0,05)$

Como se puede apreciar, la fórmula de mayor aceptación (F2), no se diferencia significativamente de las fórmulas comerciales en cuanto a los atri- 
butos relacionados con el aspecto. De los dos atributos relacionados con el olor, solo se diferencia de la hamburguesa elaborada por la Empresa 3, que presenta significativamente menor olor a ahumado que el resto. En cuanto a los atributos relacionados con el sabor, se diferenció de las hamburguesas elaboradas por las Empresas 1 y 2, (significativamente menos salada que estas, pero con el mismo nivel de sabor salado que la elaborada por la Empresa 3). Por último, en relación a la textura, en general el comportamiento fue similar al de las hamburguesas comerciales.

La Tabla 5 muestra los resultados de las determinaciones químicas y microbiológicas realizadas a la fórmula F2. La muestra analizada cumple con los requisitos bromatológicos y microbiológicos establecidos según la norma INEN 1338: 2012. El índice de costos de materias primas muestra un valor relativamente elevado, pero acorde a la calidad intrínseca del producto.

Tabla 5. Características de calidad de la hamburguesa seleccionada (F2)

\begin{tabular}{|c|c|c|c|}
\hline ANÁLISIS & UNIDAD & PARÁMETROS & MEDIA \pm DS \\
\hline \multirow[t]{4}{*}{ Químicos } & (\%) & Grasa total & $3,4 \% \pm 0,41$ \\
\hline & & Proteínas totales $(\mathrm{N} \mathrm{x} 6,25)$ : & $26,5 \% \pm 0,57$ \\
\hline & & Humedad & $\begin{array}{l}67,46 \quad \% \quad \pm \\
0,48\end{array}$ \\
\hline & & Cenizas & $0,97 \% \pm 0,02$ \\
\hline \multicolumn{4}{|l|}{ Microbiológicos } \\
\hline & $\mathrm{UFC} / \mathrm{g}$ & Aerobios mesófilos & $1 \times 101$ \\
\hline & & Escherichia Coli & $<1 \times 101$ \\
\hline & & Staphylococcus aureus & $<1 \times 101$ \\
\hline & $\mathrm{spp} / 25 \mathrm{~g}$ & Salmonella & No detectado \\
\hline Económico & $\$ / \mathrm{kg}$ & Índice de costo & 9,57 \\
\hline
\end{tabular}

Los valores son la media de 3 determinaciones.

\section{CONCLUSIONES}

Se desarrolló una formulación de hamburguesa a base de carne de ganado caprino con adición de Perejil (Petroselinum crispum) y Albahaca (Ocimum basilicum), aceptable e inocua para el consumidor, que además cumple con los estándares requeridos por la NTE INEN 1338: 2012 PARA CARNES Y PRODUCTOS CÁRNICOS, en lo referente a Productos Cárnicos Precocidos Congelados

\section{REFERENCIAS BIBLIOGRÁFICAS}

Aréchiga, C. F., Aguilera, J. I., Rincón, R. M., De Lara, S. M., Bañuelos, V. R., \& Meza-Herrera, C. A. (2008). Situación actual y perspectivas de la producción caprina ante el reto de la globalización. Tropical and Subtropical Agroecosystems, 9(1), 1-14. 
Banerjee, R., Verma, A. K., Das, A. K., Rajkumar, V., Shewalkar, A. A., \& Narkhede, H. P. (2012). Antioxidant effects of broccoli powder extract in goat meat nuggets. Meat science, 91(2), 179-184.

Das, A. K., Rajkumar, V., Verma, A. K., \& Swarup, D. (2012). Moringa oleiferia leaves extract: a natural antioxidant for retarding lipid peroxidation in cooked goat meat patties. International Journal of Food Science \& Technology, 47(3), 585-591.

Das, A. K., \& Rajkumar, V. (2013). Effect of different fat level on microwave cooking properties of goat meat patties. Journal of food science and technology, 50(6), 1206-1211.

Ilavarasan, R., Abraham, R. J., Rao, V. A., Pandiyan, V., Babu, R. N., \& Ruban, S. W. (2015). Effect of Age on Physico-Chemical and Nutritional Composition of Indigenous Kodiadu Goat Meat. Journal of Animal Research,5(3), 585.

Jalal, H., Para, P. A., Ganguly, S., Padhy, A., Praveen, P. K., \& Wakchaure, R. (2015). FAT REPLACERS IN MEAT: A BRIEF REVIEW. World Journal of Engineering Research and Technology, 1(2), 16-21.

Leite, A., Rodrigues, S., Pereira, E., Paulos, K., Oliveira, A. F., Lorenzo, J. M., \& Teixeira, A. (2015). Physicochemical properties, fatty acid profile and sensory characteristics of sheep and goat meat sausages manufactured with different pork fat levels. Meat science, 105, 114-120.

Lopes, L. S., Martins, S. R., Chizzotti, M. L., Busato, K. C., Oliveira, I. M., Neto, O. M., \& Ladeira, M. M. (2014). Meat quality and fatty acid profile of Brazilian goats subjected to different nutritional treatments. Meat Science,97(4), 602-608.

Malekian, F., Khachaturyan, M., Gebrelul, S., \& Henson, J. F. (2014). Composition and fatty acid profile of goat meat sausages with added rice bran. International journal of food science. Volumen 2014:1-8.

Malekian, F., Khachaturyan, M., Gebrelul, S., \& Henson, J. (2016). Nutritional characteristics and consumer acceptability of sausages with different combinations of goat and beef meats. Functional Foods in Health and Disease,6(1), 42-58.

Montossi, F., Font-i-Furnols, M., del Campo, M., San Julián, R., Brito, G., \& Sañudo, C. (2013). Producción sostenible de carne ovina y las tendencias en las preferencias de los consumidores: compatibilidades, contradicciones y dilemas sin resolver. PRODUCCIÓN DE CARNE OVINA DE CALIDAD, 1.

Prieto, M., Mouwen, J. M., Puente, S. L., \& Sánchez, A. I. C. (2008). Concepto de calidad en la industria agroalimentaria. Interciencia: Revista de ciencia y tecnología de América, 33(4), 258-264.

Webb, E. C. (2014). Goat meat production, composition, and quality. Animal Frontiers, 4(4), 33-37.

Williams, P. (2007). Nutritional composition of red meat. Nutrition \& Dietetics,64(s4), S113-S119.

Yadav, S., Malik, A. K., Sharma, D. P., \& Mehta, N. (2014). Influence of vacuum tumbling and particle size on quality characteristics of goat meat rolls. Asian Journal of Dairy \& Food Research. 33 (4): 302-306. 